\title{
Identification of quantifiers related to circadian rhythm variations
}

\author{
Tristan BONARGENT ${ }^{* \dagger}$, Eric PIGEON ${ }^{\dagger}$, Tristan MARTIN ${ }^{\ddagger}$, Mathieu POULIQUEN ${ }^{\dagger}$, \\ Olivier GEHAN ${ }^{\dagger}$, Tomas MENARD ${ }^{\S}$, Mohammed M'SAAD ${ }^{\dagger}$ \\ $\dagger$ Normandie Univ, UNICAEN, ENSICAEN, LAC, 14000 Caen, France \\ $\ddagger$ Normandie Univ, UNICAEN, INSERM, COMETE, 14000 Caen, France \\ $\S$ Mechatronics Department, University of Technical Education, Rajamangala University of Technology Thanyaburi,Thailand \\ * Corresponding author, e-mail: tristan.bonargent@unicaen.fr
}

\begin{abstract}
The aim of this study is to propose quantifiers allowing to characterize the adaptation speed of the circadian rhythm of a living being based on temperature measurements. While the most commonly used model to represent circadian rhythm is the COSINOR model, it is not adapted to analyze changes. An extended COSINOR model is then proposed here in order to take into account temperature disturbances related to the activity of the studied living being. The quantifiers are obtained in a two step process. First, the extended COSINOR parameters are determined with a modified recursive least square algorithm, which is modified in order to compensate for the lack of persistent excitation. The estimates of these parameters are further filtered in order to provide several quantifiers which provide useful information on the adaptation speed of the studied living being. The proposed methodology is evaluated on rats temperature measurements obtained from a laboratory experiment.
\end{abstract}

Index Terms-Circadian rhythm, least square algorithm, COSINOR, jet lag, hypergravity, rats.

\section{INTRODUCTION}

The circadian rhythm reflects the temporal organization of the biology and behavior of every life forms to anticipate and optimally deal with predictable variation of their environment [1]. The day/night variations induced by Earth rotation contribute to their acclimation by synchronizing the circadian rhythm on a period of 24 hours [2]. The circadian rhythm promotes wake state to optimally conduct our daily activity during the active time, and promotes restoration and repair during rest time. Indeed, it has a great influence on the behavior, the physical conditions, the cognitive performance, the muscular power, the sleep or even the perception of human being and some animals [3]. The easiest physiological variable allowing to measure and to identify the circadian rhythm of a living being is its internal temperature [2].

Nowadays, to ensure the best physical performances of night shift workers [4] or to speed up the recovery due to jet lag disturbances [5], the synchronization of the circadian rhythm has become an important topic. In particular, it is important to determine if it is possible to modify the adaptation speed of the circadian rhythm with external stimuli after a disturbance like jet lags. For that reason, several quantifiers are required in order to estimate the speed and time of resynchronization of the circadian rhythm after stimuli.

Several approaches have been considered in the literature in order to analyze the circadian rhythm, by using the internal temperature, as the frequency approach using periodograms, the statistical method or some methods from the field of signal processing [6]. The most popular approach is probably the Lomb and Scargle algorithm [7] which is used to compute the periodicity of the cycle with a frequency approach. It uses a sliding window that can filter out variations in high-frequency signals but does not provide any parameters other than periodicity. A statistical analysis, called ANOVA, is employed to determine the parameters of the circadian cycle. The ANOVA method is a post-processing tool with does not work in real-time. In contrast, a recursive least square algorithm, from the field of signal processing, can be used to fit the circadian rhythm in real-time with a model [8]. One of the most commonly used models to represent circadian rhythm, with this approach, is the COSINOR. Unfortunately, this purely sinusoidal model cannot take into account disturbances coming from the external environment, which leads to a bias on the results. To the authors best knowledge, no study explicitly incorporates external disturbances into the model, and are then able to provide accurate quantifiers for the adaptation speed of the circadian rhythm from external stimuli.

In this article, we propose an algorithm that provides, in real-time, quantifiers giving informations on the adaptation speed of the studied living being, namely, the resynchronization time and the speed of convergence and delay. An extended COSINOR model, taking into account external disturbances, is first proposed, whose parameters are estimated in real-time by using a modified least square algorithm that permits to give unbiased estimation of the parameters even if the persistent excitation condition is not always met. The quantifiers related to the dynamic of the circadian rhythm variations are then calculated in real-time by filtering the parameters estimates. 
The paper is organized as follows. The proposed algorithm, providing estimation of the considered quantifiers, is presented in section II. In section III, the experiment conducted, by the COMETE laboratory, on rats, is detailed. The proposed approach is then applied on the measurements obtained from the experiment, in section IV, and an interpretation of the results, in order to validate the proposed quantifiers, is given. Finally section $\mathrm{V}$ concludes the paper.

\section{Methodology}

In this section, one describes the algorithm which estimates some quantifiers related to the speed of the circadian rhythm adaptation when facing perturbations. First, the extended COSINOR model and the corresponding modified least square algorithm are presented in subsection II-A. Then, the considered quantifiers are presented in subsection II-B together with an estimation algorithm which extracts characteristic informations on the circadian rhythm adaptation.

\section{A. Estimation of circadian rhythm parameters}

\section{1) Reminder on the circadian rhythm:}

The circadian rhythm is a biological rhythm that can be described as a repetition of oscillations of a physical variable. The oscillations occur periodically. This phenomenon can be characterized with physical quantities as shown on Fig. 1 and enumerated in the following paragraph:

- the MESOR (Midline Estimating Statistic Of Rhythm) is the mean value of the signal during one period.

- the Amplitude is the half of the difference between the maximum value and the minimum value of the signal.

- the Period is the length of one oscillation.

- Acrophases are the instants corresponding to the maximum values of the signal contrary to the Batyphases which are the instants corresponding to the minimum values.

Internal temperature measurements are usually analyzed using the COSINOR model [9]. The parameters can be estimated with a recursive least square algorithm. The aim of this algorithm is to determine the set of parameters that minimizes the quadratic error between the internal temperature of the subject and the output of the model.

Since the COSINOR model only considers stable circadian rhythm, we present next an extended COSINOR model that takes into account the effects of an external stimuli on the circadian rhythm and then the internal temperature.

2) Extended COSINOR model:

The COSINOR model [8] is conventionally defined as follows:

$$
\Theta(t)=M+A \cos (2 \pi f t+\varphi)
$$

with $\Theta(t)$ the temperature, $M$ the mesor, $A$ the amplitude, $\varphi$ the phase, $f$ the frequency and $t$ the discrete time. In this study, the frequency $f$ is considered to be known with a value of $1 / 24$ hours $^{-1}$. In the classical case, the least

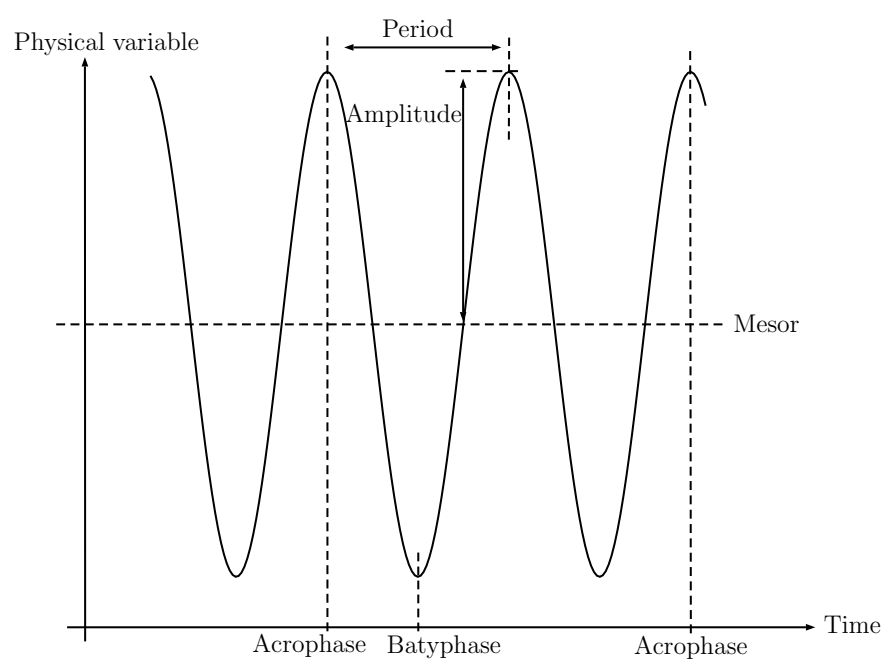

Fig. 1. Representation of the circadian rhythm

square algorithm has to give a consistent estimation of $M$, $A$ and $\varphi[10]$.

To improve the model, two signals $v(t)$ and $e(t)$, representing respectively the external stimuli and other nonmodeled inputs, are introduced. The extended COSINOR is hence defined as follows:

$\Theta(t)=M+A \cos (2 \pi f t+\varphi)+B\left(q^{-1}\right) v(t)+C\left(q^{-1}\right) e(t)$

with $B\left(q^{-1}\right)=b_{0}+b_{1} q^{-1}+b_{2} q^{-2}+\cdots+b_{n_{b}} q^{-n_{b}}$, $C\left(q^{-1}\right)=1+c_{1} q^{-1}+c_{2} q^{-2}+\cdots+c_{n_{c}} q^{-n_{c}}, n_{b}$ and $n_{c}$ represent respectively the order of $B\left(q^{-1}\right)$ and $C\left(q^{-1}\right) . q^{-1}$ is the shift operator such that $q^{-1} x(t)=x(t-1)$.

The signal $e(t)$ is assumed to be a white noise that represents the effects of the external environment on the internal temperature and $v(t)$ is a centered binary signal that represents the presence of a stimuli.

As the cosine function is not a linear combination of the parameters, it is linearized as follows :

$$
\begin{aligned}
\Theta(t)=M+\alpha \cos (2 \pi f t) & +\rho \sin (2 \pi f t) \\
& +B\left(q^{-1}\right) u(t)+C\left(q^{-1}\right) e(t)
\end{aligned}
$$

with $\alpha=A \cos (\varphi)$ and $\rho=A \sin (\varphi)$.

The parameter vector $\theta$ and the regression vector $\phi(t)$ are respectively defined by:

$$
\begin{aligned}
& \theta=\left[\begin{array}{lllllllll}
M & \alpha & \rho & b_{0} & b_{1} & \ldots & c_{1} & c_{2} & \ldots
\end{array}\right] \\
& \phi^{T}(t)=\left[\begin{array}{lllll}
1 & \cos (2 \pi f t) & \sin (2 \pi f t) & v(t) & \ldots
\end{array}\right. \\
& \left.v\left(t-n_{b}\right) \quad e(t-1) \quad \ldots \quad e\left(t-n_{c}\right)\right]
\end{aligned}
$$

Using the previous definitions, equation (3) can be re-written under the following matrix standard form :

$$
\Theta(t)=\phi^{T}(t) \theta+e(t)
$$


3) Adaptation of the recursive least square algorithm:

The vector $\hat{\theta}(t)$ containing the estimated parameters is defined as follows

$$
\hat{\theta}(t)=\left[\begin{array}{lllllllll}
\hat{M} & \hat{\alpha} & \hat{\rho} & \hat{b_{0}} & \hat{b_{1}} & \ldots & \hat{c_{1}} & \hat{c_{2}} & \ldots
\end{array}\right]
$$

The signal $e(t)$ cannot be measured since it corresponds to un-modeled inputs, as a consequence, $e(t-i)$ is replaced by the a posteriori error $\varepsilon(t-i / t-i)$, which is defined as follows

$$
\varepsilon(t-i / t-i)=\Theta(t-i)-\hat{\phi}^{T}(t-i) \hat{\theta}(t-i)
$$

This leads to the following definition of the substituted regression vector $\hat{\phi}(t)$

$$
\begin{gathered}
\hat{\phi}^{T}(t)=\left[\begin{array}{llllll}
1 & \cos (2 \pi f t) & \sin (2 \pi f t) & v(t) & \ldots & v\left(t-n_{b}\right) \\
\ldots & \varepsilon(t-1 / t-1) & \ldots & \varepsilon\left(t-n_{c} / t-n_{c}\right)
\end{array}\right]
\end{gathered}
$$

The least square algorithm proposed in this study is defined as follows [11], [12]:

$$
\left\{\begin{aligned}
\varepsilon(t / t-1) & =\Theta(t)-\hat{\phi}^{T}(t) \hat{\theta}(t-1) \\
\delta(\varepsilon(t / t-1)) & =\left\{\begin{array}{c}
0 \text { if }|\varepsilon(t / t-1)|<\gamma_{w} \\
1-\frac{\gamma_{w}}{|\varepsilon(t / t-1)|} \text { otherwise }
\end{array}\right. \\
\Gamma(t) & =\frac{\delta(\varepsilon(t / t-1)) F(t) \hat{\phi}(t)}{1+\delta(\varepsilon(t / t-1)) \hat{\phi}^{T}(t) F(t) \hat{\phi}(t)} \\
\widehat{\theta}(t) & =\widehat{\theta}(t-1)+\Gamma(t) \varepsilon(t / t-1) \\
F(t+1) & =(1-\beta)\left(I_{n}-F(t) \hat{\phi}^{T}(t)\right) F(t)+\beta F_{0}
\end{aligned}\right.
$$

with $n=3+n_{b}+n_{c}+1, \beta \in \mathbb{R}^{+}$relatively small that respect $0<\beta<1, \gamma_{w}$ an infimum of the imperfection of the model and $F_{0}$ the initial value of the matrix $F(t)$ which is chosen very large.

This algorithm is a robust modification of the standard extended least square algorithm. It permits to ensure an unbiased convergence of the parameters even if the persistent excitation conditions are not always met [13].

The role of $\delta(\varepsilon(t / t-1))$ is to freeze the adaptation if the a priori error is below the threshold $\gamma_{w}$. In the other case, $\delta(\varepsilon(t / t-1))$ progressively increases while the error increases until it approaches 1 . In this least square algorithm, the matrix $F(t)$ is computed with a filtering action controlled by $\beta$.

\section{B. Estimation of the quantifiers}

The aim of the present work is to determine characteristic factors of the resynchronization of the circadian rhythm after a disturbance. These factors are obtained from the phase $\varphi$ of the circadian rhythm. Indeed, this variable seemed to be the best physical quantity to perceive the adaptation. Therefore, two methods are proposed to compute the factors. The first one estimates the resynchronization time by estimating the convergence time of the phase $\varphi$. The second one estimates two factors: a speed of convergence and a delay corresponding to the time before the beginning of the phase shift.

1) Resynchronization time approach:

The resynchronization time can be obtained from an estimation of the derivative of the phase. A Luenberger observer [14] is implemented based on the following design model that contains the phase and its first derivative as state variables

$$
\left\{\begin{aligned}
x(t+1) & =A x(t) \\
\Theta(t) & =C x(t)
\end{aligned}\right.
$$

with $A=\left[\begin{array}{cc}1 & T_{e} \\ 0 & 1\end{array}\right], C=\left[\begin{array}{ll}1 & 0\end{array}\right], x(t)=\left[\begin{array}{ll}x_{1}(t) & x_{2}(t)\end{array}\right]^{T} \in$ $\mathbb{R}^{2}$ is the state vector, $\Theta(t)=x_{1}(t) \in \mathbb{R}$ the phase and $T_{e}$ the sampling period. Please note that $x_{2}(t)$ represents the first derivative of $\Theta(t)$ and hence the signal to be estimated.

Based on the model (10), the Luenberger observer that provides a consistent estimation $\hat{x}(t)=\left[\begin{array}{ll}\hat{x}_{1}(t) & \hat{x}_{2}(t)\end{array}\right]^{T}$ of $x(t)$ is given by

$$
\hat{x}(t+1)=A \hat{x}(t)-K(\hat{\Theta}(t)-\Theta(t))
$$

where the gain $K \in \mathbb{R}^{2 \times 1}$ is calculated to adjust the eigenvalues of the matrix $(A-K C)$ in the stability domain and to ensure a compromise between the speed of convergence and the observer sensitivity to the noise measurement.

The time of resynchronization is inferred form a threshold calculated from the derivative of the phase. Indeed, the value of the threshold is computed from the maximum of the derivative of the phase between the interval $\left[t_{1}, t_{2}\right]$, with $t_{1}$ corresponds to the time of convergence of the least square algorithm and $t_{2}$ the time of the stimuli.

The convergence time is finally obtained by computing the last instant for which the derivative of the phase is above the threshold. The resynchronization time corresponds to the convergence time minus the perturbation time. This method is illustrated Fig. 2.

2) Speed of convergence and delay approach:

The second method provides an estimation of the speed of convergence to the new rhythm and the delay taken to begin the phase shift. This is done by fitting on the estimated phase a second order model with a delay and a zero. The equation of the model is given in equation (12).

$$
G(s)=K_{p} \frac{-T_{z}+s}{1+\frac{2 \zeta}{\omega_{0}} s+\left(\frac{s}{\omega_{0}}\right)^{2}} e^{-T_{d} s}
$$

with $K_{p}$ the static gain, $\zeta$ the damping, $\omega_{o}$ the natural frequency, $T_{d}$ the delay that is related to the time taken before the shift and $T_{z}$ the zero.

In this model, the natural frequency $\omega_{o}$ is related to the bandwidth and thus to the speed of convergence and $T_{d}$ to the time taken before the beginning of the phase shift. The zero $T_{z}$ was added to obtain a better fit of the model. In fact, the zero in the frequency domain doesn't disturb the second order model because of its small value. 

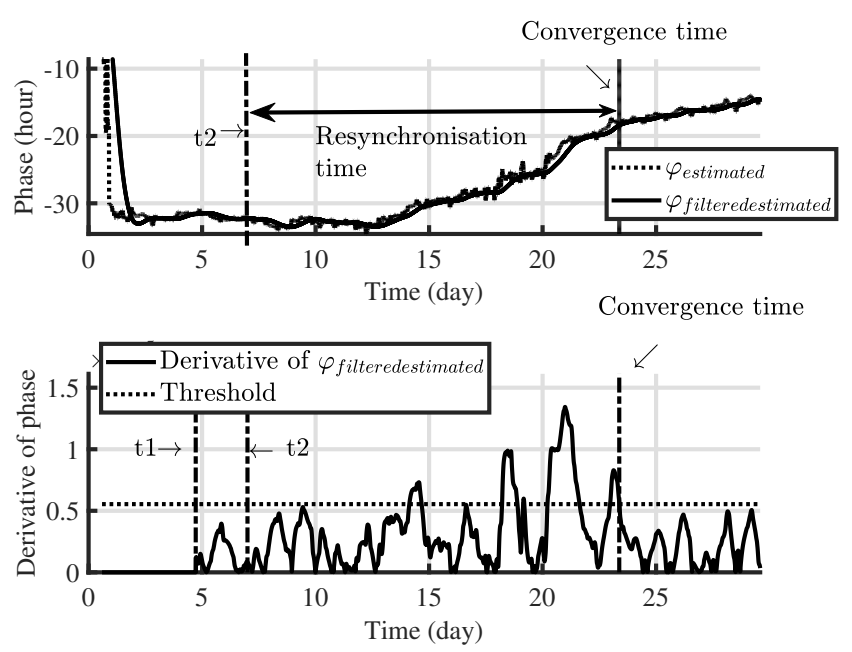

Fig. 2. Estimation of the time of convergence

The curve is considered as a step response of a delayed second order model as illustrated on Fig. 3. This second method consists in identifying the delay and the natural frequency of this model.
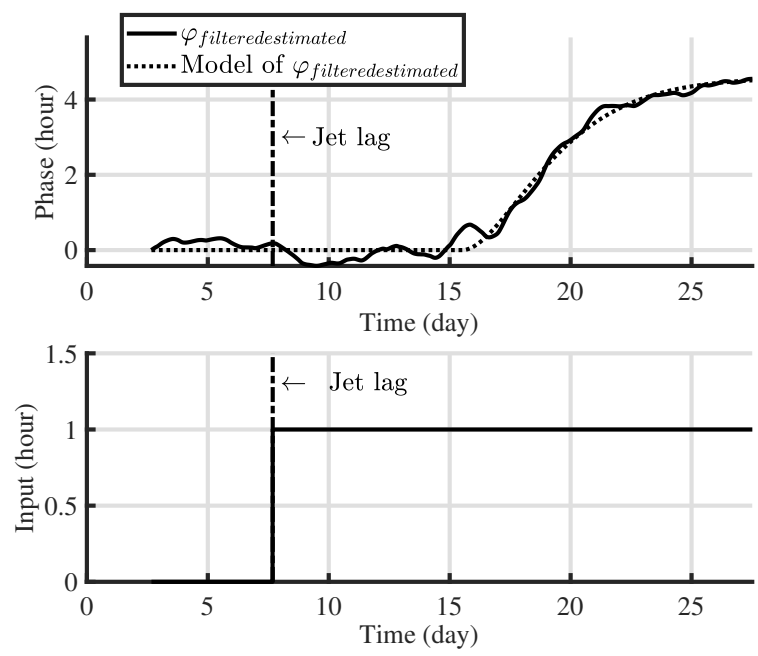

Fig. 3. Estimation of a model: Jet lag (SHAM and BVL) and Jet lag plus $2 \mathrm{G}$ (only BVL)

\section{DESCRIPTION OF THE EXPERIMENT}

Besides from light information, the vestibular system plays an important role in the synchronization of circadian rhythms [7]. It is an organ that sens every head acceleration in all directions and thus gives sense of balance and gravity. The vestibular system is sensitive and can be affected with external perturbations. An experiment was conducted on rats to highlight the effects of jet lags and vestibular stimulation by hypergravity on circadian rhythms adaptation to jet lag. This experiment was set up in the COMETE UMR-S 1075 INSERM laboratory.
The vestibular system plays an important role in the circadian rhythm [15]. It is a sensor that gives a spatial orientation and a sense of balance. The vestibular system is sensitive and can be affected by external perturbations. Research is being carried out to determine factors that can modify the rate of adaptation of this rhythm. An experiment was conducted on rats to highlight the effects of jet lags and hypergravity on circadian rhythm. This experiment was set up in the COMETE UMR-S 1075 INSERM laboratory of CAEN University .

Two different groups of rats were used. The first one was called bilateral vestibular loss (BVL) induced using an injection of chemicals products. The perception of movement by the inner ear was then abolished permanently. The second group was called SHAM. This group underwent the same operation than the BVL but were injected with a placebo.

The two groups of rats were separated in three sub-groups. Each sub-group endured different perturbations. The three sub-groups are called JET LAG, 2G and JET LAG+2G. Their protocols are described as follow:

1) JET LAG: This first group was exposed to a 6 hours jet lag. This was done by changing the time of the cycle Day-Night of the experiment room. The rats were used to have their lights turning off at 8 p.m. When the perturbation began, the darkness occurred at 2 p.m. It is a phase advance of 6 hours.

2) $2 \mathrm{G}$ : The rats of the $2 \mathrm{G}$ group underwent a hypergravity representing the double of the earth gravity. The material used was a centrifuge for small animal. This was done once a day at a fixed hour during the Acrophase.

3) JET LAG+2G: The rats in this last group endured the two types of perturbations exposed in 1) and 2) at the same time.

The physical quantity acquired during the experiment was the internal temperature of the rats. Thermometers contained in pills were given to the rodents to monitor the temperature. It was measured 7 days before the perturbation and until the end of the experiment. 14 rats were used in each subgroup and each sub-group was divided by two with BVLs and SHAMs.

\section{RESULTS OF THE EXPERIMENTATION}

\section{A. Implementation of the selected algorithm}

The presented algorithm has been used on data corresponding to experiments conducted by the COMETE laboratory. The different aspects that need to be considered are described in the following.

The signal $v(t)$ that represents the stimuli is defined here as the centrifuge impact on the rats behavior. The signal, before being centered, takes the value 1 when the centrifuge is on and 0 otherwise. The signal $e(t)$ represents here other perturbations corresponding to various activities, such as for example when the rats eat.

The threshold of the first method of quantification is increased by $40 \%$ to have a suitable estimation of the resynchronization time for all rats. 
The input of the model is different for the three groups because of the nature of the perturbation. It is described in the following paragraph.

1) Jet lag (SHAM and BVL) and Jet lag $+2 \mathrm{G}$ (only BVL) For this group, the model is computed after the convergence time $t_{1}$ of the least square algorithm. The input was set to 0 before the time perturbation time $t_{2}$ and to 1 after. This is illustrated on Fig. 3.

2) $2 \mathrm{G}$ and Jet lag $+2 \mathrm{G}$ (only SHAM)

For these groups, no model was calculated due to the impact of the centrifuge on the phase.

The algorithm has been validated to ensure the unbiased convergence of estimated parameters for this experiment with the autocorrelation rate of the residuals [13].

The results of the proposed methodology applied to the rat experiment are now presented. All results are given with their mean values and their standard deviations .

1) JET LAG group (BVL)

The rats in this group were subject to an 8 hours JET LAG. The phase evolves progressively to the new referenced hour, after a delay. The mean bandwidth is $0.34(0.17) \mathrm{rad} / \mathrm{s}$, the resynchronization time is $15.65(3.29)$, and the delay is 6.73 (2.48) days. The evolution of the phase for BVLs and SHAMs is presented below on Fig. 4.

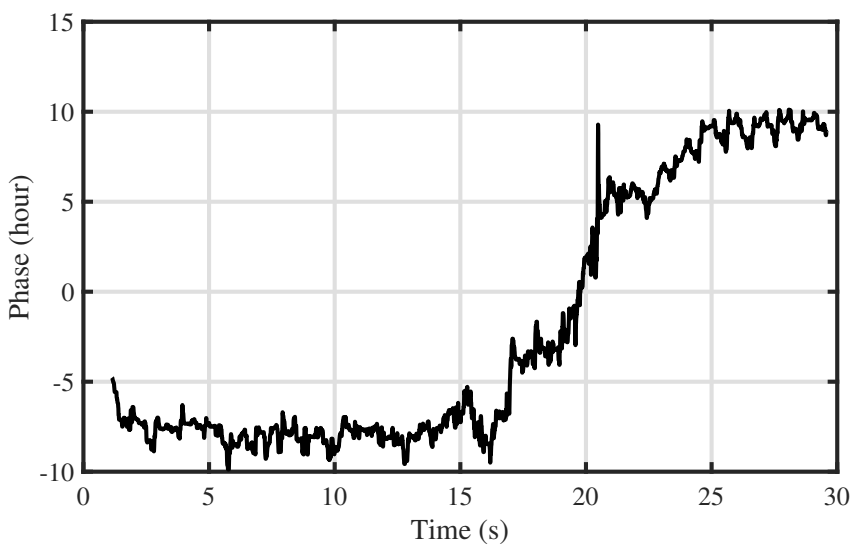

Fig. 4. Evolution of the phase of the JET LAG group during the experiment (BVL)

\section{2) $2 \mathrm{G}$ group (SHAM)}

In this group, the rats were placed in a centrifuge once a day for one hour, during one week. SHAMs see their phase falling during the centrifuge time. Their resynchronization time are 8.92 (2.09) days thus they take 1.92 days to stabilize their phase after the last centrifuge Fig. 5.

3) JET LAG+2G group (BVL)

This group experience a jet lag of 8 hours and a centrifuge at the same time. The BVLs have the same variation as the JET LAG group. Thus, the BVLs seems to not feel the centrifuge as the SHAM. They have a bandwidth of $0.24(0.05) \mathrm{rad} / \mathrm{s}$, a delay of 5.67 (0.95) days and a resynchronization time of 17.02 (3.30) days.

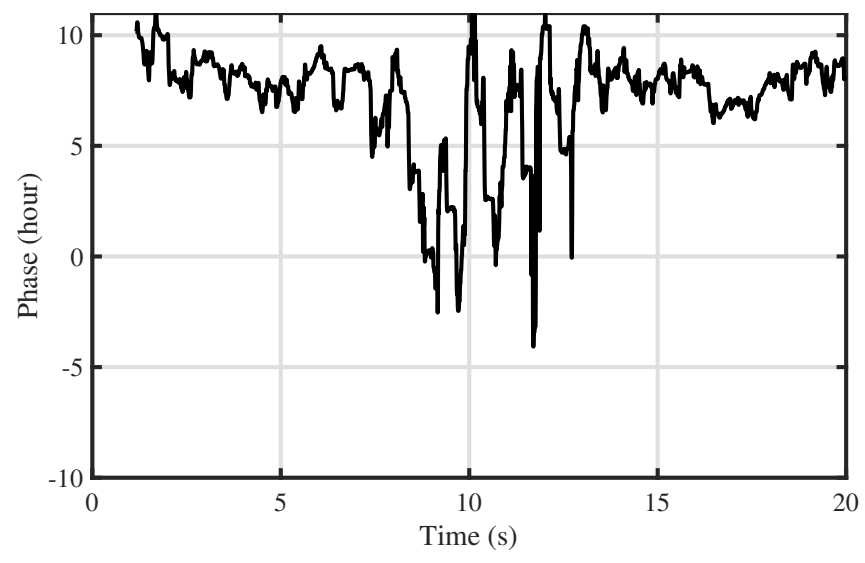

Fig. 5. Evolution of the phase of the $2 \mathrm{G}$ group during the experiment. (SHAM)

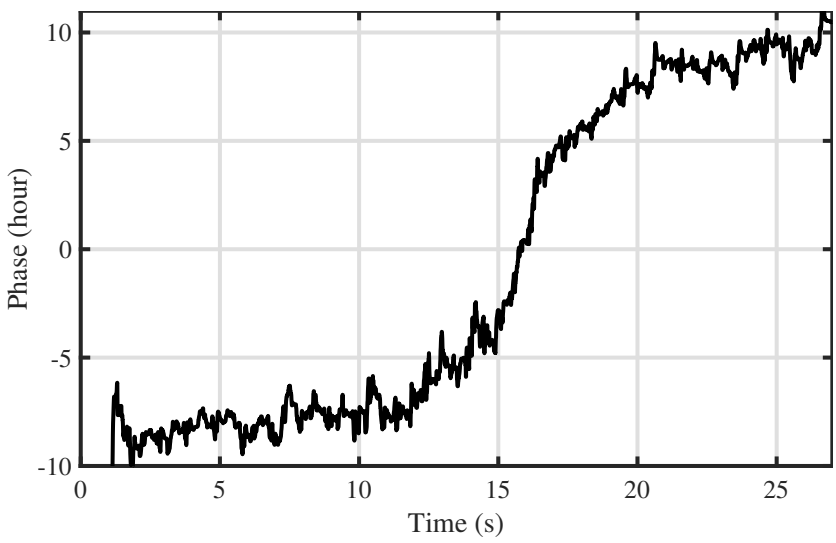

Fig. 6. Evolution of the phase of the JET LAG+2G group during the experiment (BVL)

The results are summarized in the Table I. A comparison between the BVL of the first group and the last group shows that the rats of the third group take less time to begin their phase shift but their speed of convergence is lower than the first group.

\begin{tabular}{|l|c|}
\hline JET LAG group & BVL \\
Bandwidth (rad/s) & $0.34(0.17)$ \\
Delay (day) & $6.73(2.48)$ \\
Resynchronization time (day) & $15.65(3.29)$ \\
\hline \hline 2G group & SHAM \\
Bandwidth (rad/s) & $/(/)$ \\
Delay (day) & $/(/)$ \\
Resynchronization time (h) & $8.92(2.09)$ \\
\hline \hline JET LAG+2G group & BVL \\
Bandwidth (rad/s) & $0.24(0.05)$ \\
Delay (day) & $5.67(0.95)$ \\
Resynchronization time (day) & $17.02(3.30)$ \\
\hline
\end{tabular}

TABLE I

QUANTIFIERS RELATED TO CIRCADIAN RHYTHM VARIATIONS

\section{CONCLUSION}

The problem of defining quantifiers characterizing the dynamics of circadian rhythm variations has been considered 
in this paper. To this purpose, an extended COSINOR model has been proposed to take into account stimuli and external sources of disturbance. Its parameters have been estimated with a modified Least Square Algorithm that is less sensitive to the lack of persistent excitation. Based on the estimated parameters, two quantification methods have been set up to assess the dynamics of the phase. The first one, based on the phase derivative, allows to estimate a time of resynchronization. The second one allows to estimate a convergence speed, considering the phase as a step response of a second order model with a delay and a zero. Finally, the proposed method has been tested on data issued from experiments performed on rats under specific conditions.

\section{REFERENCES}

[1] A. Reinberg, M. H. Smolensky, M. Riedel, Y. Touitou, N. Le Floc'h, R. Clarisse, M. Marlot, S. Berrez, D. Pelisse, and B. Mauvieux, "Chronobiologic perspectives of black time-Accident risk is greatest at night: An opinion paper," Chronobiology International, vol. 32, no. 7, pp. 1005-1018, 2015.

[2] R. Refinetti, "The circadian rhythm of body temperature," Frontier in Bioscience (Landmark Edition), vol. 15, pp. 564-594, Jan. 2010.

[3] T. Reilly and B. Edwards, "Altered sleep-wake cycles and physical performance in athletes," Physiology \& Behavior, vol. 90, no. 2, pp. 274-284, Feb. 2007.

[4] H. J. Burgess, K. M. Sharkey, and C. I. Eastman, "Bright light, dark and melatonin can promote circadian adaptation in night shift workers," Sleep Medicine Reviews, vol. 6, no. 5, pp. 407-420, Oct. 2002.

[5] C. H. Samuels, "Jet Lag and Travel Fatigue: A Comprehensive Management Plan for Sport Medicine Physicians and High-Performance Support Teams," Clinical Journal of Sport Medicine, vol. 22, no. 3, p. 268, May 2012

[6] R. Refinetti, G. Cornélissen, and F. Halberg, "Procedures for numerical analysis of circadian rhythms," Biological Rhythm Research, vol. 38, no. 4, pp. 275-325, Aug. 2007.

[7] N. R. Lomb, "Least-squares frequency analysis of unequally spaced data," Astrophysics and Space Science, vol. 39, no. 2, pp. 447-462, Feb. 1976 .

[8] D. S. Hickey, J. L. Kirkland, S. B. Lucas, and M. Lye, "Analysis of circadian rhythms by fitting a least squares sine curve," Computers in Biology and Medicine, vol. 14, no. 2, pp. 217-223, 1984.

[9] F. Halberg, Y. L. Tong, and E. A. Johnson, "Circadian System Phase - An Aspect of Temporal Morphology; Procedures and Illustrative Examples," in The Cellular Aspects of Biorhythms. Springer, Berlin Heidelberg, 1967, pp. 20-48.

[10] E. Pigeon, J.-B. Fabin, M. Pouliquen, B. Mauvieux, O. Gehan, T. Ménard, A. Goudjil, and S. Moussay, "Identification of circadian rhythm," Jun. 2016.

[11] M. Pouliquen, E. Pigeon, and O. Gehan, "Identification Scheme for Hammerstein Output Error Models With Bounded Noise," IEEE Transactions on Automatic Control, vol. 61, no. 2, pp. 550-555, Feb. 2016.

[12] M. M'Saad, Adaptation Paramétrique aux moindres carrés, ENSICAEN Cours de troisième année.

[13] L. Ljung, System Identification: Theory for the User. Prentice Hall PTR, 1999.

[14] D. Luenberger, "An introduction to observers," IEEE Transactions on Automatic Control, vol. 16, no. 6, pp. 596-602, Dec. 1971.

[15] P. M. Fuller and C. A. Fuller, "Genetic evidence for a neurovestibular influence on the mammalian circadian pacemaker," Journal of Biological Rhythms, vol. 21, no. 3, pp. 177-184, Jun. 2006. 